\title{
Fast neutron inelastic scattering from ${ }^{7} \mathbf{L i}$
}

Roland Beyer ${ }^{1, \star}$, Axel Frotscher ${ }^{1,2, \dagger}$, Arnd R. Junghans ${ }^{1}$, Markus Nyman ${ }^{3}$, Arjan Plompen ${ }^{3}$, Marcel Grieger ${ }^{1,2}$, Toni Kögler ${ }^{1,2}$, Felix Ludwig ${ }^{1,2}$, Stefan Reinicke ${ }^{1,2}$, Stefan Schulz ${ }^{1,2}$, Ronald Schwengner ${ }^{1}$, Thu Trang Trinh ${ }^{1}$, Sebastian $\mathrm{Urla}^{1,2}$, and Andreas Wagner ${ }^{1}$

${ }^{1}$ Helmholtz-Zentrum Dresden - Rossendorf, 01328 Dresden, Germany

${ }^{2}$ Technische Universität Dresden, 01062 Dresden, Germany

${ }^{3}$ European Commission, Joint Research Centre, 2440 Geel, Belgium

\begin{abstract}
The inelastic scattering of fast neutrons from ${ }^{7} \mathrm{Li}$ nuclei was investigated at the $n$ ELBE neutrontime-of-flight facility. The photon production cross section of $478 \mathrm{keV} \gamma$-rays from the first excited state of ${ }^{7} \mathrm{Li}$ was determined by irradiating a disc of $\mathrm{LiF}$ with neutrons of energies ranging from $100 \mathrm{keV}$ to about $10 \mathrm{MeV}$. The target position was surounded by a setup of $7 \mathrm{LaBr}_{3}$ scintillation detectors and 7 high-purity germanium detectors to detect the de-excitation $\gamma$-rays. A ${ }^{235} \mathrm{U}$ fission chamber was used to determine the incoming neutron flux. The number of detected photons was corrected for the detection efficiency, multiple scattering and the time-of-flight dependent data acquisition dead time. The preliminary results show reasonable agreement with some previous measurments but are about $15 \%$ below the recent data taken at the GELINA facility.
\end{abstract}

\section{Introduction}

Inelastic neutron scattering on ${ }^{7} \mathrm{Li}$ has technological implications in fusion and fission reactors. In the former it could create an intense $\gamma$-ray field causing heating and radiation damage, in the latter it could strongly influence the neutron energy spectrum and therefore the neutronics of e.g. novel reactor concepts like the molten salt reactor.

Furthermore the $\gamma$-ray production cross section of ${ }^{7} \mathrm{Li}$ could be used as an alternative for neutron fluence determination to enable relative measurements of neutron-induced reactions [1]. Inelastic neutron scattering on ${ }^{7} \mathrm{Li}$ leads to the production of a $478 \mathrm{keV} \gamma$-ray from the first excited state of ${ }^{7} \mathrm{Li}$. The next higher lying state in this nucleus at $4630 \mathrm{keV}$ already undergoes break up into an $\alpha$-particle and a triton. The angular distribution of the $\gamma$-rays after inelastic neutron scattering is isotropic and has negligible internal conversion. The threshold energy of $546 \mathrm{keV}$ is low enough to be able to cover a large range of neutron energies. The cross section of about 0.2 barn has only little and fairly smooth structures. It is reasonably high to enable good statistics within a feasible measurement time. With these properties ${ }^{7} \mathrm{Li}$ seems to be a good candidate to replace for instance neutron induced fission of ${ }^{235} \mathrm{U}$ as reference cross section especially in short or low neutron flux experiments.

These possible applications of lithium require a detailed knowledge of the inelastic neutron scattering cross section with low uncertainty. Nyman et al. [2] compiled and compared the available data and revealed rather large

\footnotetext{
^e-mail: roland.beyer@hzdr.de

†Present address: Technische Universität Darmstadt, 64277 Darmstadt, Germany
}

discrepancies. In Fig. 1 all available data sets with more than 10 data points in the energy region from threshold till $20 \mathrm{MeV}$ are plotted (references can be found in [2]). The latest measurement was done by Nyman et al. at the GELINA facility in Geel, Belgium, and was published in 2016 [2]. It was measured at two different flight paths. Both data sets agree well with the data from Presser et al. at least up to $4 \mathrm{MeV}$. But these data are about $10 \%$ above the data set from Morgan et al., which in turn is again somewhat higher than what Smith, Olsen and Freeman measured. At $2 \mathrm{MeV}$ the experimental data are discrepant by up to $25 \%$. Also the data sets from evaluated databases differ by up to $5 \%$. CENDL which coincides with JENDL mainly relies on the Morgan data while ENDF which is equivalent to JEFF obviously included also the lower lying data sets. Therefore up to now this cross section is far from being able to be used as a standard. To add some more information and to check the results of Nyman et al. a measurement was performed at the $n$ ELBE neutron time-of-flight facility.

\section{The $n$ ELBE neutron time-of-flight facility}

The neutron time-of-flight (ToF) facility $n$ ELBE is the first photo-neutron source at a superconducting electron accelerator, which allows a very precise time structure of the neutron pulses and also favorable background conditions due to the low instantaneous neutron flux and the absence of any moderating materials. At $n$ ELBE an electron beam of about $30 \mathrm{MeV}$ kinetic energy is focused onto a liquid lead target to produce bremsstrahlung that subsequently produces neutrons via photo-nuclear reactions on the lead nuclei. The short (5 ps) micropulse length and variable 


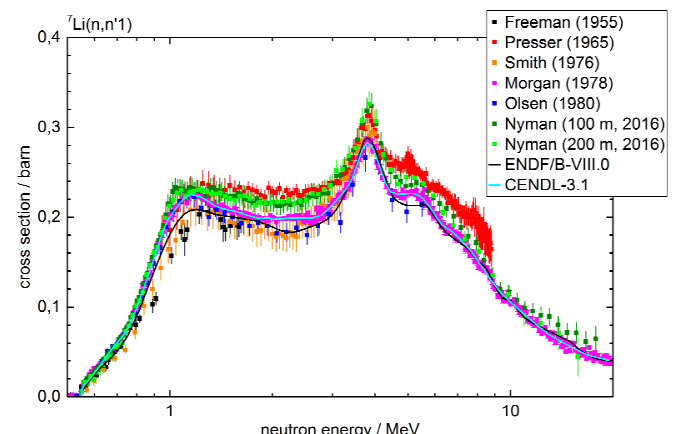

Figure 1. Currently available cross section data for ${ }^{7} \operatorname{Li}\left(n, n^{\prime} \gamma\right)$. Data taken from [2] and references therein.

repetition rate (typically $25-400 \mathrm{kHz}$ ) of the electron beam of the ELBE accelerator $[3,4]$ together with a very compact neutron producing target (thickness $11 \mathrm{~mm}$ ) [5] allows to use a short flight path (5 to $11 \mathrm{~m}$ ) and a correspondingly high neutron intensity. With such a neutron producing target the energy resolution in the fast neutron range is dominated by the achievable ToF resolution of the detectors used. The response functions of different neutron ToF facilities are discussed in detail in the review article by Schillebeeckx et al. [6].

The $n$ ELBE neutron spectrum ranges from about 10 $\mathrm{keV}$ up to $10 \mathrm{MeV}$. The source strength is typically around $2 \cdot 10^{11} \mathrm{n} / \mathrm{s}$ which scales down to a neutron flux of approximately $3 \cdot 10^{4} \mathrm{n} / \mathrm{cm}^{2} / \mathrm{s}$ at the sample position. Further properties of the $n$ ELBE neutron beam are described in detail in Ref. [7].

\section{The experimental setup}

In the experimental area separated by a $2.5 \mathrm{~m}$ thick wall from the neutron producing target the detector setup was placed as shown in Fig. 2. The incoming neutron beam shaped by a collimator in the wall first passed a ${ }^{235} \mathrm{U}$ fission chamber. This fission chamber (called H19 and provided by the PTB Braunschweig, Germany) [8, 9] contains a total mass of $201 \mathrm{mg}{ }^{235} \mathrm{U}$ and it was placed at a flight path of $6 \mathrm{~m}$ from the neutron producing target. Behind the fission chamber, the neutrons hit the sample, which consisted of two LiF disks of $80 \mathrm{~mm}$ diameter and $2 \mathrm{~mm}$ thickness each (the same samples as used in Ref. [2]), at a flight path of $8.3 \mathrm{~m}$. In a plane around the sample position several photon detectors were placed: Seven high purity germanium (HPGe) detectors and seven $\mathrm{LaBr}_{3}$ scintillation detectors. The HPGe detectors had $60 \%$ relative efficiency and were placed at angles of 90, 125 and 150 degrees relative to the incoming beam. Six of the HPGe detectors are combined in two threefold clusters with a common cooling system but separated readout. $\mathrm{The}_{\mathrm{LaBr}}$ detectors were three 3 in $\times 3$ in and four 2 in $\times 2$ in scintillators placed at angles between 30 and 150 degrees as indicated in Fig. 2. All detectors had distances between 22 to $30 \mathrm{~cm}$ from the sample.

For each detector time and energy information was acquired by a VME based data acquisition system.

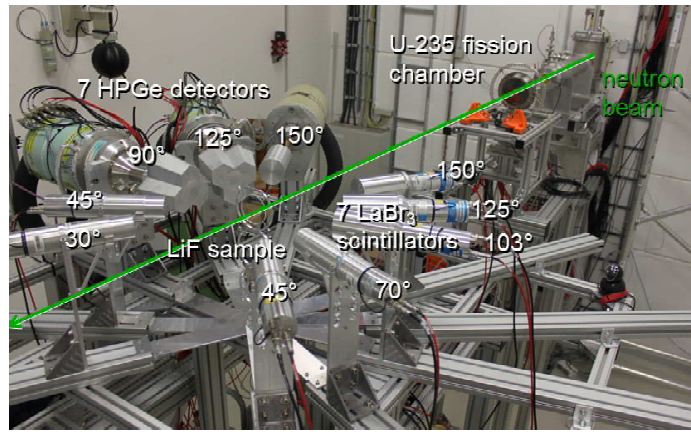

Figure 2. The experimental setup.

\section{Determination of the $\gamma$-ray production cross section}

From the acquired data the photon production cross section was deduced using the following formula:

$$
\sigma=\frac{N_{\text {det }}}{N_{\mathrm{n}} \cdot f_{\text {mult }} \cdot n_{\mathrm{T}} \cdot \eta_{\text {live }} \cdot \varepsilon}
$$

where $N_{\text {det }}$ is the number of detected photons, $N_{\mathrm{n}}$ the neutron fluence at the position of the fission chamber including dead time correction, $f_{\text {mult }}$ the correction for neutron transmission to the sample and multiple scattering inside the sample, $n_{\mathrm{T}}$ the areal density of ${ }^{7} \mathrm{Li}$ inside the target, $\eta_{\text {live }}$ a ToF-dependent dead time correction factor, and $\varepsilon$ the efficiency of the detector including a correction for extended source geometry. $n_{\mathrm{T}}$ can be calculated from the geometry of the sample, molar volume and the isotopic abundance that can be found in databases. The other quantities will be explained below.

$\eta_{\text {live }}$ is caused by the fact that the data acquisition was not able to register a neutron event while it is busy with the conversion of an event that is caused by the so called photon flash, i.e. the bremsstrahlung also emitted from the neutron producing target. This ToF dependence can be determined by measuring the dead time value for each single event and folding the ToF distribution with that [7]. Two separated data acquisition systems were used, one for the $\mathrm{LaBr}_{3}$ detectors and one for the HPGe detectors and the fission chamber. This results in the two different dead time distributions that are ploted in Fig. 3 over the uncalibrated ToF. The quantity $\alpha(T)$ is the ratio of live-to-real time $t_{\text {live }}(T) / t_{\text {real }}(T)$ for each single ToF bin $T$. From the $\alpha$ values and the measurement real time for each single detector and the one of the fission chamber one can calculate the dead time correction factors $\eta_{\text {live }}$ according to:

$$
\eta_{\text {live,det }}=\frac{\alpha_{\text {det }}}{\alpha_{\mathrm{FC}}} \cdot \frac{t_{\text {real,det }}}{t_{\text {real,FC }}}
$$

where the index "det" denotes one of the HPGe or $\mathrm{LaBr}_{3}$ detectors.

The neutron fluence $N_{\mathrm{n}}$ is calculated from the ToF spectrum of the fission chamber after applying a cut on the pulse height to remove events from alpha activity and a correction for the fragment detection efficiency. After conversion to a neutron energy scale and the includsion of the IAEA standard ${ }^{235} \mathrm{U}$ fission cross section and the areal 


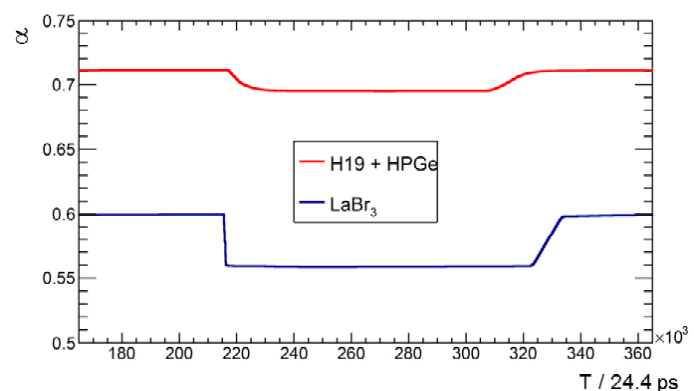

Figure 3. ToF dependent dead time of the two data acquisition systems.

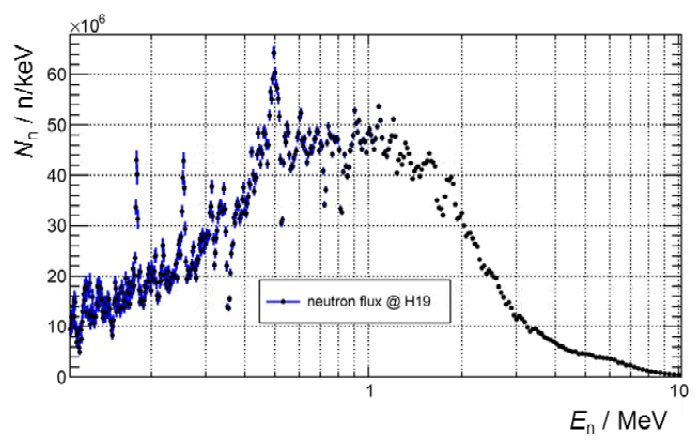

Figure 4. Neutron fluence determined from the ${ }^{235} \mathrm{U}$ fission chamber data.

density of fissile nuclei, the neutron fluence shown in Fig. 4 is obtained.

$N_{\mathrm{n}}$ is the number of neutrons that were arriving at the position of the fission chamber. To get the number of neutrons that hit the sample a correction has to be applied that has to contain the transmission through part of the material of the fission chamber and through the air between that chamber and the sample. Furthermore the effect of attenuation, moderation and multiple scattering inside the sample has to be estimated. To get this correction factor the Monte-Carlo code MCNP6 [10] was used. In the simulation a realistic neutron spectrum and the detailed geometry was implemented. The effects of multi-scattering and the loss of time-to-energy correlation is included by using the so called track length estimator tally type 4 available in MCNP and weighting the flux in the sample with the energy dependent inelastic scattering cross section of ${ }^{7} \mathrm{Li}$ taken from ENDF/B-7.1. Finally, the correction is determined from the ratio of the simulated neutron flux in the sample with a filled geometry relative to a void geometry. The result is plotted in Fig. 5 showing a correction $f_{\text {mult }}$ of up to $10 \%$ for these effects.

The detection efficiencies $\varepsilon$ of all detectors was determined using calibrated point sources of ${ }^{88} \mathrm{Y},{ }^{133} \mathrm{Ba},{ }^{137} \mathrm{Cs}$ and ${ }^{152} \mathrm{Eu}$. The discrepancy between the calibration measurement using point sources and the measurement with an extended neutron beam incident onto an extended LiFsample was estimated by Monte-Carlo simulation using Geant4 [11]. A comparison of the number of photons entering each detector when using a point source with the case of an volume source revealed a correction of about 3 to $5 \%$. The efficiency value at $E_{\gamma}=478 \mathrm{keV}$ was deter-

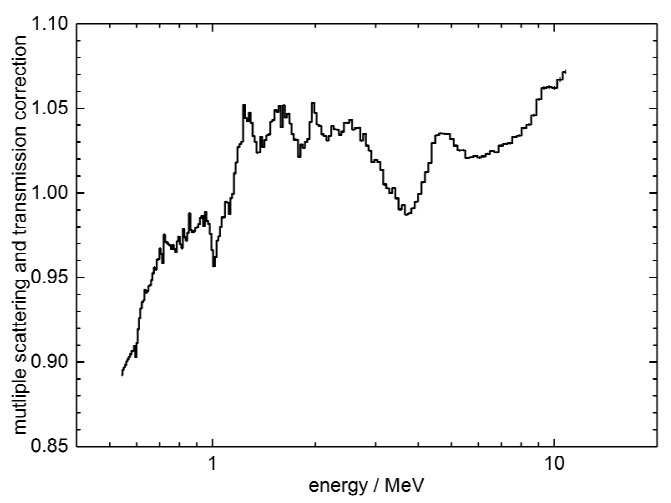

Figure 5. Correction factor accounting for neutron transmission from the fission chamber to the sample and moderation and multiple scattering inside the sample.

mined by interpolation using an empirical function fitted to the efficiency values at the photon energies emitted by the calibrated sources. The resulting values for $\varepsilon$ range from 0.8 to $3.6 \times 10^{-3}$ and 0.7 to $1.0 \times 10^{-3}$ for the $\mathrm{LaBr}_{3}$ and HPGe detectors, respectively. Due to the uncertainties of the activities of the calibrated sources and the interpolation procedure $\Delta \varepsilon$ is in the order of $2.5 \%$.

The numbers of detected photons $N_{\text {det }}$ were determined from 2D histograms as shown in Fig. 6. There detected photon energy is plotted against the neutron ToF. The $478 \mathrm{keV}$ line from the de-excitation of the ${ }^{7} \mathrm{Li}$ first excited state is clearly visible. The next line above is the 511 $\mathrm{keV}$ line from the annihilation of positrons created by the photon flash in air. The HPGe detectors can resolve these two lines, while for the $\mathrm{LaBr}_{3}$ they are hard to separate. To get the neutron energy resolved cross section these 2D plots were cut into time slices and the number of $478 \mathrm{keV}$ photons for each time-of-fligh bin was determined. For the HPGe detectors this was done by determining a constant background level and summing all events above this level inside the region of interest around the $478 \mathrm{keV}$ peak. Additionally also the evolution of the intensity of the $511 \mathrm{keV}$ peak was examined to determine the lifetime of positrons in air, which came out to be 116(7) ns. This information was used to calculate the area of the $511 \mathrm{keV}$ peak in the spectra from the $\mathrm{LaBr}_{3}$ detectors. Furthermore the position and the width for that peak was fixed to improve the fitting procedure for the $478 \mathrm{keV}$ peak.

The resulting photon production cross sections calculated via Eq. (1) are shown in Fig. 7 for each detector used. One can see that the agreement within each detector group is reasonabe well. To compare the two detector types with each other and also with the previous measurements and evaluations, mean cross sections were calculated for the HPGe detectors and for the $\mathrm{LaBr}_{3}$, respectively (see Fig. 7 bottom). The results of the present work are in reasonable agreement with the ENDF evaluation and slightly lower than CENDL but by about $15 \%$ lower than the data from Geel. Fig. 7 contains only statistical uncertainties. The systematic uncertainties sum up to about $4 \%$, where the largest contribution is caused by the determination of the 

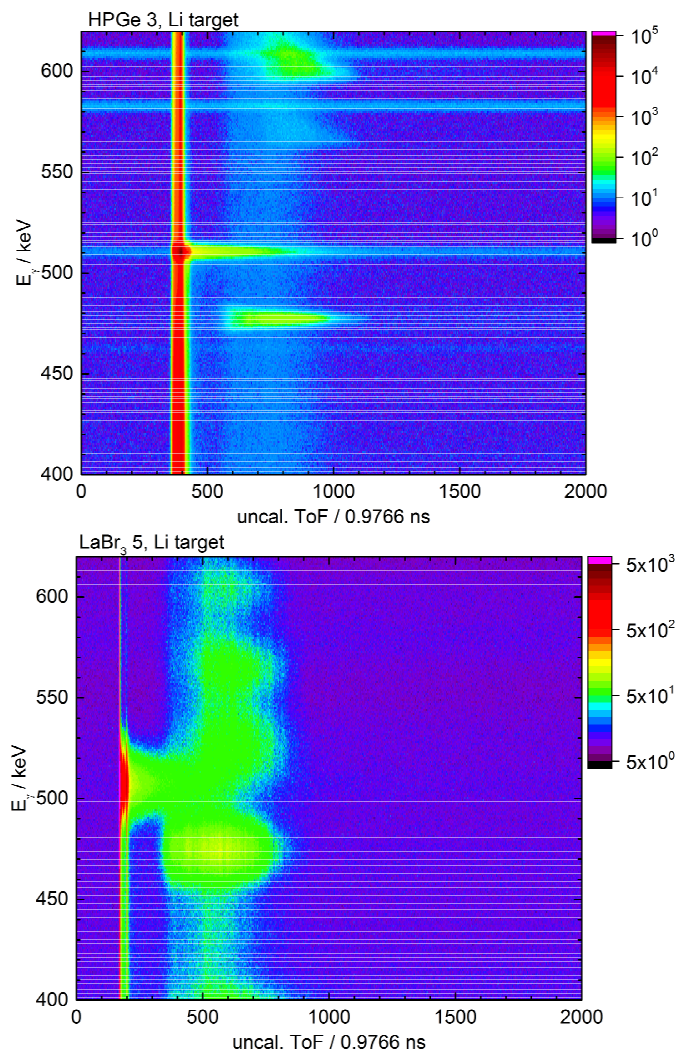

Figure 6. Energy vs. ToF spectra for a HPGe (top) and a $\mathrm{LaBr}_{3}$ detector (bottom).

photon detection efficiency mainly due to the interpolation to $478 \mathrm{keV}$ energy.

\section{Summary and Outlook}

At the neutron ToF facility $n$ ELBE the inelastic scattering on ${ }^{7} \mathrm{Li}$ was investigated. The inelastic scattering cross section for the excitation of the $E_{\mathrm{x}}=478 \mathrm{keV}$ state was determined via a $\gamma$-ray production measurement. The results of the $\gamma$-ray production measurement agree with data from many previous experiments and evaluations. But a clear deviation (ca. $15 \%$ ) to the most recent measurement performed at the GELINA facility was found.

Therefore, a second measurement was performed recently at $n$ ELBE with a few improvements: To enable the determination of $\varepsilon$ exactly at $478 \mathrm{keV}$ a ${ }^{7} \mathrm{Be}$ source was produced via proton irradiation of a small LiF disk. Vacuum tubes were installed to reduce the intensity of the 511 $\mathrm{keV}$ peak and the data acquisition systems were improved. The data analysis of that measurement is still ongoing.

\section{Acknowledgement}

We thank Ralf Nolte for providing the ${ }^{235} \mathrm{U}$ fission chamber. We thank the staff of the ELBE accelerator for their cooperation during the experiments and A. Hartmann, K. Heidel and M. Görler for technical assistance. This work was supported by the European Commission within the CHANDA project (FP7-605203).
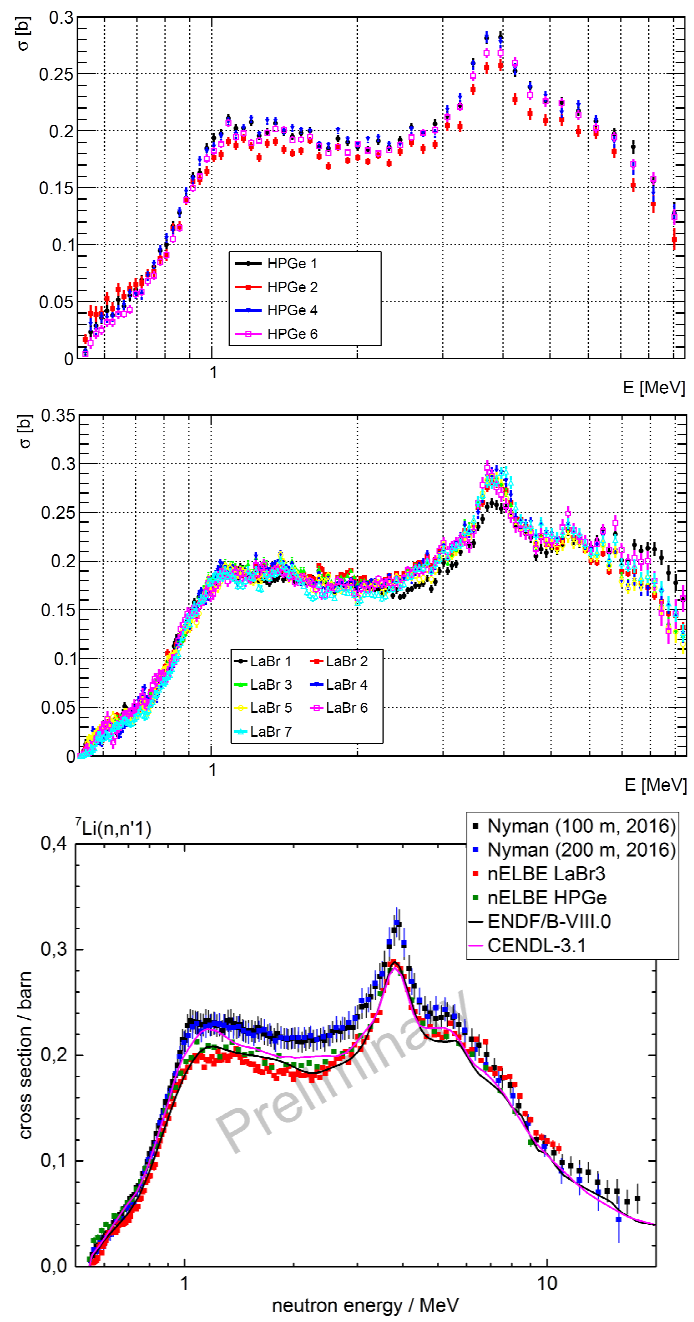

Figure 7. Preliminary photon production cross section for the ${ }^{7} \mathrm{Li} 478 \mathrm{keV} \gamma$-ray determined by each single HPGe (top) and $\mathrm{LaBr}_{3}$ (middle) detector. The bottom graph compares measured mean values from the present work with previous measurements [2] and evaluations.

\section{References}

[1] A.D. Carlson et al., Nucl. Data Sheets 118, 126 (2014)

[2] M. Nyman et al., Phys. Rev. C 93, 024610 (2016)

[3] F. Gabriel et al., Nucl. Instr. Meth. B 161, 1143 (2000)

[4] J. Teichert et al., Nucl. Instr. Meth. A 507, 354 (2003)

[5] E. Altstadt et al., Ann. Nucl. Ene. 34, 36 (2007)

[6] P. Schillebeeckx et al., Nucl. Data Sheets 113, 3054 (2012)

[7] R. Beyer et al., Nucl. Instr. Meth. A 723, 151 (2013)

[8] D.B. Gayther, Metrologia 27, 221 (1990)

[9] R. Nolte et al., Nucl. Sci. Eng. 156, 197 (2007)

[10] J.F. Briesmeister, Los Alamos National Laboratory Report LA13709 (2000)

[11] S. Agostinelli et al., Nucl. Instr. Meth. A 506, 250 (2003) 